DOI: 10.17533/udea.efyd.v33n1a10

URL DOI: http://doi.org/10.17533/udea.efyd.v33n1a10

\title{
NIVELES DE ACTIVIDAD FÍSICA DE NIÑOS Y ADOLESCENTES DURANTE EL DESCANSO EN LA ESCUELA, UN ESTUDIO OBSERVACIONAL CON EL USO DE SOPLAY
}

\author{
NÍVEIS DE ATIVIDADE FÍSICA DE CRIANÇAS \\ E ADOLESCENTES DURANTE OS INTERVALOS \\ NA ESCOLA, UM ESTUDO OBSERVACIONAL COM O USO \\ DE SOPLAY
}

\section{CHILDREN AND ADOLESCENTS PHYSICAL ACTIVITY LEVELS DURING SCHOOL BREAK, AN OBSERVATIONAL STUDY USING SOPLAY}

\section{Elkin Alberto Arias Arias}

Doctor en Ciencias del Deporte por Georg-August-Universit t

Profesor e Investigador del Grupo de Investigaci n en Ciencias Aplicadas a la Actividad F sica y el Ejercicio (GRICAFDE) del Instituto de Educaci n F sica de la Universidad de Antioquia (Colombia)

elkinariasde@googlemail.com

Arias A., E. A. (2014). Niveles de actividad fsica de ni os y adolescentes durante el descanso en la escuela, un estudio observacional con el uso de soplay. Revista Educaci n F sica y Deporte, 33 (1), 175-191, Ene-Jul 2014

\section{RESUMEN}

Marco te rico: La pr ctica de Actividad F sica (AF) en la escuela es muy importante para los ni os de hogares de bajos ingresos ubicados en zonas deprimidas. Estos ni os no pueden tener acceso a programas extraescolares de AF debido a la falta de ofertas adecuadas en la zona, los problemas de seguridad, por estar en situaci $\mathrm{n}$ de ni o trabajador o por falta de recursos 
financieros (Blatchtford, Baines y Pellegrini, 2003). La escuela parece ser un lugar apropiado para moverse durante el descanso. Sin embargo, no est claro c mo los factores ambientales en el patio de la escuela influyen en los niveles de AF de ni os con edades diferentes. Tambi $n$ existen importantes diferencias en la AF de los ni os y adolescentes. La necesidad innata de moverse puede contribuir a los niveles de AF $\mathrm{m}$ s altos en los ni os que en los de los adolescentes, incluso en ambientes similares (Rowland \& Hughes, 2006). Objetivo: En este estudio se compararon los niveles de actividad f sica de los ni os y adolescentes que comparten la misma escuela (los adolescentes van a la escuela por la ma ana, los ni os van por la tarde). $M$ todo: Los observadores utilizaron el sistema SOPLAY (System for Observing Play and Leisure Activity in Youth) (McKenzie, 2000) para obtener datos sobre la cantidad y el tipo de AF que practican los ni os y adolescentes durante los descansos. Resultados: Los resultados indican que el $52,7 \%$ de los estudiantes no realizan ning $n$ tipo de AF durante el reposo y permanecen sedentarios, el 27,2\% practica AF de moderada intensidad, el 20,1\% practica AF vigorosa. 47,3\% de los escolares y adolescentes practican actividad $\mathrm{f}$ sica de moderada a vigorosa intensidad (MVPA). Los resultados muestran diferencias significativas $(p<0,5)$ entre los ni os y adolescentes, los ni os son $\mathrm{m}$ s activos durante el receso escolar que los adolescentes.

PALABRAS CLAVE: Actividad f sica, sedentarismo, ni os, escuela

\section{RESUMO}

Marco te rico: a pr tica de Atividade F sica (AF) na escola muito importante para as crianças de fam lias com poucos recursos situadas em reas desfavorecidas. Estas crianças n o podem ter acesso a programas extraescolares de AF devido falta de ofertas adequadas na rea, os problemas de segurança, por estar em situaç o de menor trabalhador ou por falta de recursos financeiros (Blatchtford, Baines e Pellegrini, 2003). A escola parece ser um lugar apropriado para movimentar-se durante o intervalo. Por $\mathrm{m}, \mathrm{n}$ o est claro como os fatores ambientais no $\mathrm{p}$ tio da escola influenciam nos $\mathrm{n}$ veis de AF de crianças com idades diferentes. Tamb m existem importantes diferenças na AF das crianças e adolescentes. A necessidade inata de movimentar-se pode contribuir aos $n$ veis de AF mais altos nas crianças que nos adolescentes, inclusive em ambientes similares (Rowland \& Hughes, 2006). Objetivo: Neste estudo compararamse os $n$ veis de atividade $f$ sica das crianças e adolescentes que dividem a mesma escola (os adolescentes $\vee \mathrm{o}$ escola de manh, as crianças $\vee \mathrm{o}$ tarde). $M$ todo: Os observadores utilizaram o sistema SOPLAY (System for Observing Play and Leisure Activity in Youth) (McKenzie, 2000) para 
obter dados sobre a quantidade e o tipo de AF que praticam as crianças e adolescentes durante os intervalos. Resultados: Os resultados indicam que $52,7 \%$ dos estudantes n o realizam nenhum tipo de AF durante o repouso

e permanecem sedent rios, $27,2 \%$ pratica AF de intensidade moderada, $20,1 \%$ pratica AF vigorosa. $47,3 \%$ das crianças e adolescentes praticam atividade $f$ sica de intensidade moderada a vigorosa (MVPA). Os resultados mostram diferenças significativas $(p<0,5)$ entre as crianças e adolescentes, as crianças s o mais ativas durante o intervalo escolar que os adolescentes.

PALAVRAS-CHAVE: Atividade f sica, sedentarismo, crianças, escola

\section{ABSTRACT}

Theoretical background: The physical activity (PA) practice in a school is very important for children from low-income households located in depressed areas. The children cannot have access to extra-curricular PA programs due to the lack of appropriate offers for these areas, security problems for being working children or the lack of financial resources (Blatchtford, Baines \&Pellegrini, 2003). School seems to be an appropriate place to be active at break time. However, it is not clear how environmental factors at school yard influence the children PA levels at different ages. In addition, there are important differences between children and adolescents related to PA. Children's innate necessity to move can contribute to increase their PA levels compared to adolescents even in similar environments (Rowland \& Hughes, 2006). Objective: in this study children and adolescents PA levels from the same school were compared (adolescents go to school in the morning, children go in the afternoon). The observers used the SOPLAY system (System for Observing Play and Leisure Activity in Youth) (McKenzie, 2000) to obtain the data related to the quantity and type of PA developed by children and adolescents during the schools breaks. Results: the results indicate that $52.7 \%$ of the students do not perform any type of PA during the schools breaks and remain sedentary, 27.2\% practice PA in a moderate intensity, 20,1\% perform PA with a strong intensity. $47,3 \%$ of school children and adolescents perform PA from moderate to vigorous intensity (MVPA). The results show significant differences $(p<.5)$ between children and adolescents, children are more active during school breaks than adolescents.

KEY WORDS: Physical activity, extra-curricular programs, sedentary, children, adolescents, school, recess 


\section{INTRODUCCIÓN}

La inactividad $f$ sica est asociada con numerosas enfermedades que, sin embargo, son prevenibles, y es reconocida como un serio problema de salud p blica. El Departamento de Salud y Servicios Humanos de Estados Unidos recomienda a los nios y adolescentes de 6 a 17 a os participar en 60 minutos, o $\mathrm{m} \mathrm{s}$, diarios, de actividad $\mathrm{f}$ sica, desde moderada hasta vigorosa (MVPA por sus siglas en ingl s) (USDHHS, 2008). La actividad $f$ sica (en adelante AF) practicada por los ni os y adolescentes deber a, adem s, ser apropiada a su nivel de desarrollo, divertida, incluir actividades variadas y la cantidad de minutos recomendados pueden ser obtenidos en varios periodos de actividad acumulados durante el d a (USDHHS \& USDA, 2005).

Diferencias en g nero (los ni os y los $\mathrm{j}$ venes son $\mathrm{m}$ s activos que las ni as y las $\mathrm{j}$ venes), y edad (los ni os son $\mathrm{m}$ s activos que los adolescentes), han sido reportados (Vilhjalmsson \& Kristjansdottir, 2003). Sin embargo, a n no est clara la influencia del ambiente en la disminuci $\mathrm{n}$ de los niveles de la actividad $\mathrm{f}$ sica con la edad. Existen tambi $\mathrm{n}$ importantes diferencias en la AF de ni os y adolescentes; la AF de los ni os consiste en intermitentes y espont neas explosiones de actividad y los ni os no se ocupan de la misma actividad por largo tiempo. Los adolescentes tienen mayor autonom a y prefieren actividades organizadas (Pangrazi, 2000). Diferencias en los niveles de AF se podr an presentar debido a variables ambientales, psicol gicas o sociol gicas (McKenzie, Sallis \& Elder, 1997). En este estudio se analizan ambos grupos separadamente con consideraci $\mathrm{n}$ de la variable ambiental.

Numerosas instituciones gubernamentales de salud y estudios cient ficos sugieren que las escuelas deben cumplir un papel proactivo en la promoci $\mathrm{n}$ de la AF para prevenir problemas de salud, tales como enfermedad cardiovascular, obesidad y diabetes tipo 2. La AF en la escuela es particularmente importante 
para ni os que provienen de hogares de bajos ingresos situados en zonas deprimidas. Estos ni os pueden no tener acceso a programas de actividad $f$ sica que se ofrecen afuera de la escuela debido a la falta de oferta en la zona, a problemas de seguridad, a su necesidad de trabajar o a la falta de recursos econ micos (Blatchtford, Baines \& Pellegrini, 2003).

La mayor a de los estudios sobre AF en la escuela se enfocan en la educaci $\mathrm{n} f$ sica (en adelante EF). Sin embargo, sta rea se enfoca en objetivos de aprendizaje espec ficos, en un ambiente altamente estructurado, lo que en muchos casos no proporciona la oportunidad de practicar suficiente AF (Pate, Davis \& Robinson, 2006). Adicionalmente, la baja frecuencia con la que la EF es ofrecida en instituciones escolares no permite cumplir con las recomendaciones de 60 minutos diarios (USDHHS, 2008). Poco se conoce acerca de la pr ctica voluntaria de la AF por parte de los ni os y hay pocos estudios acerca de c mo los periodos de descanso en la escuela contribuyen a la cantidad total de AF diaria (Wechsler, Devereaux, Davis \& Collins, 2000).

Algunos estudios (Beighle, Morgan, LeMasurier \& Pangrazi, 2006; Sarkin, McKenzie \& Sallis, 1997; Mota, Suva, Santos, Ribeiro, Oliverira \& Duarte, 2005; Ridgers, Stratton, Fairclough \& Twisk, 2007; Verstraete, Cardon, DeClercq \& Bourdeaudhuij, 2006) han cuantificado la AF de los ni os en los descansos en la escuela utilizando monitores del ritmo card aco, pod metros $\mathrm{y}$ aceler metros, pero estos $\mathrm{m}$ todos proveen poca informaci $\mathrm{n}$ sobre el contexto en el cual la actividad se lleva a cabo. Por otro lado, pocos estudios han indicado qu estrategias, tales como la reestructuraci $n$ del patio de la escuela y el aumento de su atractivo trav s de la restauraci $n$ y el uso de colores (Stratton \& Mullan, 2005) y la disposici n de elementos de juego (Verstraete et al., 2006), puede ser til para incrementar la cantidad de AF de los ni-

os durante el receso. Recientemente se ha despertado el inter $\mathrm{s}$ por cuantificar la cantidad de AF desde una perspectiva ecol gica, con el uso de la observaci n directa (Stratton \& Mullan, 2005; Da- 
vison \& Lawson, 2006; McKenzie, Sallis \& Elder 1997; McKenzie, Baquero, Crespo, Arredondo, Campbell \& Elder, 2008; Ridgers, Stratton \& McKenzie, 2010). Este $\mathrm{m}$ todo tiene la ventaja de que permite registrar la cantidad de AF y, simult neamente, registrar tambi $n$ las influencias contextuales, incluyendo condiciones del ambiente $f$ sico y social (McKenzie, 2002). Evaluar la influencia de factores potencialmente modificables, y sus relaciones con la AF, es importante para el dise o de intervenciones orientadas a incrementar la cantidad de actividad $\mathrm{f}$ sica en los ni os. El prop sito del presente estudio fue usar la observaci n directa para: a) Evaluar la contribuci $n$ del descanso escolar a las metas de cantidad de AF en una escuela de nivel socio-econ mico medio-bajo, y b) Medir, de forma objetiva, la cantidad de AF en ni os de b sica primaria durante el descanso escolar y compararla con la cantidad de AF de adolescentes de secundaria.

\section{MÉTODO}

\section{Población y Muestra}

Los datos fueron obtenidos durante observaciones realizadas en una escuela $\mathrm{p}$ blica de la zona noroccidental de la ciudad de Medell $n$ (Colombia). Esta escuela tiene la particularidad de atender j venes adolescentes de $6^{\circ}$ a $11^{\circ}$ grado (en su mayor a de 11 a 17 a os) en horas de la ma ana, y a ni os de $1^{\circ}$ a $5^{\circ}$ grado (en su mayor a de 6 a 10 a os) en las mismas instalaciones. Esta caracter stica permite la comparaci $n$ de los niveles de AF en ni os y adolescentes durante per odos de descanso que tienen lugar en los mismos espacios con las mismas estructuras y facilidades. La escuela atiende un total de 820 ni os y 952 adolescentes.

Los datos fueron recogidos en un per odo de dos meses, durante $5 \mathrm{~d}$ as en la escuela. Las observaciones fueron hechas durante per odos de descanso largo y corto. El per odo de descanso largo tiene lugar a media ma ana y a media tarde y durante esos 
per odos la mayor a de los ni os consumen los alimentos (desayuno o almuerzo); el per odo de descanso corto tiene lugar entre el descanso largo y la hora de salida. Los ni os de b sica primaria $\left(1^{\circ}\right.$ a $6^{\circ}$ grado $)$ no tienen descanso corto, pero permanecen solo 5 horas en la escuela, una hora menos que los adolescentes, quienes permanecen 6 horas.

Todas las reas potenciales para la pr ctica de AF fueron identificadas y medidas en la escuela antes de la recolecci $n$ de los datos. Los observadores discutieron y llegaron a acuerdos sobre la localizaci n, tama o y I mites de cada rea, y despu s de algunas pruebas sobre las perspectivas, acercamientos y tipo de im genes que deb a percibir cada c mara. Se elaboraron mapas detallados de cada rea y en ellos se se al el lugar en donde se deb an ubicar las c maras. Se seleccion un total de 6 reas en donde se observ que los ni os y adolescentes practicaran AF, aunque no todas las reas eran aptas para la AF (por ejemplo los corredores y las escalas). En la escuela objeto de observaci n no se dispon a de espacios internos para la pr ctica de actividad $f$ sica, tales como coliseos o aulas m Itiples; la escuela dispone de un patio peque-

$\mathrm{o}$ al aire libre y uno $\mathrm{m} \mathrm{s}$ grande, cubierto.

\section{Instrumentos}

Instrumento de observaci $n$

Los observadores utilizaron el instrumento denominado SOPLAY (System for Observing Play and Leisure Activity in Youth, Sistema para Observaci $n$ del Juego y la Actividad de Ocio en J venes); ste es un instrumento dise ado para obtener datos sobre el $\mathrm{n}$ mero de ni os y sus niveles de AF durante los descansos (McKenzie, Marshall, Sallis \& Conway, 2000). El sistema est basado en muestreos moment neos y ha sido utilizado previamente en varios estudios (McKenzie, Marshall, Sallis, \& Conway, 2000; Anthamatten, Brink, Lampe, Greenwood, Kingston \& Nigg, 2011; Springer, Tanguturi, Ranjit, Skala \& Kelder, 2009) para determinar la cantidad de AF de los ni os durante el descanso; una versi $n$ 
modificada se usa para estudiar los niveles de AF en los parques (McKenzie, Cohen, Sehgal, Williamson \& Golinelli, 2006).

Se hicieron escaneados sistem ticos de las reas de observaci $\mathrm{n}$ durante los dos per odos (descanso largo y descanso corto). Durante un escaneo, la AF de cada escolar en un rea es codificada como sedentaria (por ejemplo recostado, sentado o de pie), AF de moderada intensidad (incluyendo caminata) o AF vigorosa. Esta forma de codificar el nivel de actividad $f$ sica a partir de la observaci n, as como el instrumento SOPLAY, han sido validados en estudios que cuantifican la frecuencia card aca (McKenzie, Sallis \& Patterson, 1991) y que utilizan acelerometr a (De Saint-maurice, 2009; Ridgers, Stratton \& McKenzie, 2010).

\section{Procedimientos}

Protocolo de observaci $n$

Las observaciones fueron realizadas durante $5 \mathrm{~d}$ as en un per odo de 2 meses; las reas fueron observadas en un orden espec fico durante cada per odo de observaci n. Los momentos de observaci $\mathrm{n}$ fueron seleccionados aleatoriamente, pudiendo tener lugar la observaci $\mathrm{n}$ al minuto 5, 10, 15, 20 o 25 del descanso largo, o al minuto 5, 10 o 15 del descanso corto. Los observadores utilizaron una c mara de video para registrar las im genes sobre la AF $f$ sica de los escolares en los descansos. La lente de la c mara hac a un recorrido (escaneo) de derecha a izquierda y registraba a todos los escolares que se encontraban en ese momento en el rea. Durante la observaci $\mathrm{n}$ del video se le asign un c digo a cada escolar registrado en la imagen, el c digo represent el nivel de actividad observado y fue registrado en una planilla. Se hicieron registros separados para ni as y ni os.

Entrenamiento de los observadores y calibraci $n$ Se entrenaron 3 observadores para hacer la recolecci $\mathrm{n}$ de los datos. Ellos memorizaron las definiciones conceptuales y operacionales de las dimensiones del comportamiento y sus c digos 
y aprendieron los procedimientos generales para la recolecci $n$ de los datos. Se realizaron var as pr cticas de observaci $n$ y ensayo con y sin c maras. El entrenamiento se realiz hasta que los observadores alcanzaron una correlaci $\mathrm{n}$ inter-observador de $\mathrm{m}$ s del $80 \%$ al observar los mismos segmentos del video. Cada semana se realizaron revisiones para verificar el nivel de entrenamiento y la correlaci $\mathrm{n}$ inter-observador.

An lisis de los datos

La unidad de an lisis fue un escaneo de observaci $\mathrm{n}$ de un rea determinada. El tama o de la muestra fue de 153 escaneos. Se utilizaron par metros estad sticos descriptivos para obtener medidas de tendencia central y de dispersi $\mathrm{n}$ (proporciones, promedios y desviaciones est ndar). La hip tesis de la distribuci $n$ normal no fue rechazada al aplicar el test de Kolmogorov-Smirnov (>0.05) y la homogeneidad de las varianzas fue confirmada con el test de Fischer; por lo tanto, para estas variables se aplic la prueba $t$ de Student, para comparar los niveles de actividad $\mathrm{f}$ sica entre g neros y entre ni os y adolescentes. Se realizaron an lisis separados para cada variable dependiente (sedentarios, AF de mediana intensidad y AF de vigorosa intensidad).

Debido a la alta variabilidad en el $n$ mero total de ni os o adolescentes en las reas observadas, los datos de las observaciones fueron expresados como proporciones para representar el porcentaje de ni os en cada nivel de actividad. Esto se hizo al dividir el $\mathrm{n}$ mero de ni os o adolescentes observado entre sedentarios, medianamente activos y activos. Estas proporciones fueron calculadas de forma separada para cada g nero. Del total de ni os y adolescentes observados se obtuvieron entonces tres categor as, seg $\mathrm{n}$ la proporci $\mathrm{n}$ de ni os y adolescentes sedentarios, en AF de mediana intensidad y AF vigorosa. Una categor a adicional se cre al sumar la proporci $\mathrm{n}$ de ni os o adolescentes en AF de moderada intensidad y aquellos en AF vigorosa para obtener la proporci $n$ de sujetos en MVPA. 


\section{RESULTADOS}

Al promediar las proporciones en cada nivel de actividad del total de los escolares observados, se encuentra que $52,7 \%$ de los escolares no realizan ning $\mathrm{n}$ tipo de AF durante el descanso y permanecen sedentarios; $27,2 \%$ realizan AF de moderada intensidad; 20,1\% realizan AF vigorosa y 47,3\% de los escolares realizan actividad $f$ sica de intensidad moderada a vigorosa (MVPA). Este valor indica que menos de la mitad de los escolares utilizan el descanso para la pr ctica de la actividad f sica. La tabla 1 muestra el porcentaje promedio de escolares observados en cada nivel de AF.

\begin{tabular}{l|c}
\hline Nivel de AF & Promedio (DE) \\
\hline \% Sedentario & $52,7(26,5)$ \\
\% AF Moderada Intensidad & $27,2(16,8)$ \\
\% AF Vigorosa & $20,1(22,1)$ \\
\% MVPA & $47,3(26,5)$ \\
\hline
\end{tabular}

Tabla 1. Porcentaje promedio de niños y adolescentes en determinados niveles de AF durante los descansos en la escuela (DE Desviación Estándar; MVPA actividad física de moderada intensidad, más actividad física vigorosa).

Al hacer un an lisis diferenciado de los niveles de actividad $\mathrm{f}$ sica de ni os y adolescentes se observa que los ni os y las nias de $b$ sica primaria son $\mathrm{m}$ s activos que el promedio durante el descanso; especialmente en los ni os de g nero masculino se encuentra una mayor tendencia hacia el movimiento y menor proporci n de sedentarios durante el descanso. La tabla 2 muestra los niveles de AF de ni os y ni as.

\begin{tabular}{l|c|c|c}
\hline & $\begin{array}{c}\text { Total } \\
\text { Promedio (DE) }\end{array}$ & $\begin{array}{c}\text { Niñas } \\
\text { Promedio (DE) }\end{array}$ & $\begin{array}{c}\text { Niños } \\
\text { Promedio (DE) }\end{array}$ \\
\hline \% Sedentario & $45,4(24,1)$ & $50,9(24,3)$ & $41,7(23,8)$ \\
$\begin{array}{l}\text { \% AF Moderada } \\
\text { Intensidad }\end{array}$ & $27,7(13,2)$ & $27,4(14,1)$ & $28,0(12,6)$ \\
\hline
\end{tabular}




\begin{tabular}{l|c|c|c}
\hline & $\begin{array}{c}\text { Total } \\
\text { Promedio (DE) }\end{array}$ & $\begin{array}{c}\text { Niñas } \\
\text { Promedio (DE) }\end{array}$ & $\begin{array}{c}\text { Niños } \\
\text { Promedio (DE) }\end{array}$ \\
\hline$\%$ AF Vigorosa & $26,9(21,5)$ & $23,6(19,8)$ & $30,2(22,9)$ \\
$\%$ MVPA & $54,6(24,1)$ & $49,1(24,3)$ & $58,3(23,8)$ \\
\hline
\end{tabular}

Tabla 2. Porcentaje promedio de niños en determinados niveles de AF durante los descansos en la escuela (DE Desviación Estándar; MVPA actividad física de moderada intensidad, más actividad física vigorosa).

En los adolescentes se pueden observar niveles mucho $\mathrm{m} \mathrm{s}$ elevados de sedentarismo. La proporci $\mathrm{n}$ de quienes practican MVPA vigorosa es mucho menor en los adolescentes; la disminuci n de hasta $20 \%$ indica que, con el aumento de la edad, disminuye la tendencia al movimiento, aun cuando se tiene un per odo adicional de descanso. La tabla 3 muestra el porcentaje promedio de adolescentes que permanecen sedentarios y los que realizan AF; los valores indican un elevado sedentarismo y una marcada disminuci $\mathrm{n}$ de la proporci $\mathrm{n}$ de AF en relaci $\mathrm{n}$ con los ni os.

\begin{tabular}{l|c|c|c}
\hline & $\begin{array}{c}\text { Total } \\
\text { Promedio (DE) }\end{array}$ & $\begin{array}{c}\text { Femenino } \\
\text { Promedio (DE) }\end{array}$ & $\begin{array}{c}\text { Masculino } \\
\text { Promedio (DE) }\end{array}$ \\
\hline \% Sedentario & $65,1(26,0)$ & $66,7(23,4)$ & $62,5(29,2)$ \\
$\begin{array}{l}\text { \% AF Moderada } \\
\text { Intensidad }\end{array}$ & $27,2(21,6)$ & $28,0(20,8)$ & $25,0(22,6)$ \\
\% AF Vigorosa & $7,7(17,3)$ & $5,3(15,5)$ & $12,6(20,8)$ \\
\% MVPA & $34,9(26,0)$ & $33,3(23,4)$ & $37,5(29,2)$ \\
\hline
\end{tabular}

Tabla 3. Porcentaje promedio de adolescentes en determinados niveles de AF durante los descansos en la escuela (DE Desviación Estándar; MVPA actividad física de moderada intensidad, mas actividad física vigorosa).

En la gr fica 1 se puede observar con mayor claridad las diferencias en los niveles de AF en ni os y adolescentes durante el descanso escolar. 


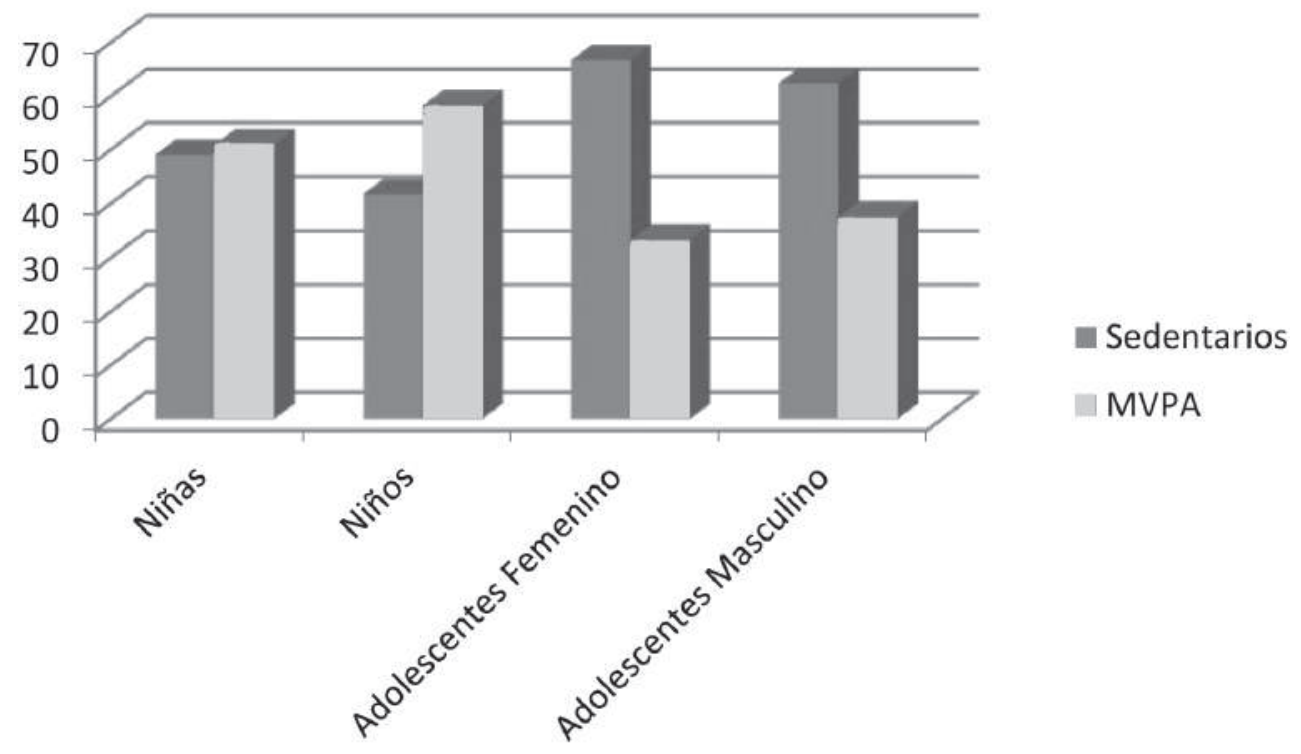

Gráfica 1. Porcentaje promedio de niños y adolescentes que permanecen sedentarios o realizan AF de mediana a vigorosa intensidad durante los descansos en la escuela.

Para las variables que indican sobre el porcentaje promedio de ni os y adolescentes sedentarios o practicando AF de intensidad moderada hasta vigorosa (MVPA) se ejecut una prueba $t$ de Student para determinar diferencias significativas entre nios y ni as, entre adolescentes femeninos y masculinos y entre ni os y adolescentes de ambos g neros. Los resultados indican diferencias significativas entre el porcentaje promedio de ni as y el porcentaje promedio de adolescentes de g nero femenino que permanecen sedentarias durante el descanso $(t[48]=-2,52$; $\mathrm{p}<.05)$; tambi $\mathrm{n}$ se encontraron diferencias significativas entre el porcentaje promedio de ni os y adolescentes de g nero masculino que permanecen sedentarios $(t[48]=-2,73 ; p<.05)$.

No se encontraron diferencias significativas al comparar el porcentaje promedio de activos y sedentarios por $\mathrm{g}$ nero $(t[60]=$ $1,20 ; p>.05)$, aunque los ni os presentan mayores niveles de actividad y menores niveles de sedentarismo. En los adolescentes tampoco se presentan diferencias de $g$ nero $(t[36]=0,49 ; p>$.05). Por 
supuesto, los ni os de g nero masculino son mucho $\mathrm{m}$ s activos y menos sedentarios durante el descanso que las adolescentes, y aunque los adolescentes son $\mathrm{m}$ s activos que las adolescentes, su nivel de sedentarismo es mayor que el de las ni as.

En general, el porcentaje promedio de ni os de ambos g neros que practican MVPA durante el descanso es mucho $\mathrm{m} \mathrm{s}$ alto que el porcentaje promedio de adolescentes $(t$ [98] $=3,72$; $\mathrm{p}<.05)$ y la proporci $\mathrm{n}$ de sedentarios es mucho $\mathrm{m}$ s baja $(t[98]$ $=-3,72 ; p<.05)$.

\section{DISCUSIÓN}

La pr ctica de AF en la escuela es de gran importancia para aquellos ni os que viven en zonas deprimidas y cuyos padres son de bajos ingresos. Debido al car cter estructurado de las clases de educaci $\mathrm{n} f$ sica, los ni os no tienen la oportunidad de practicar AF durante mucho tiempo en clase (Sarkin, McKenzie \& Sallis, 1997). El descanso parece ser apropiado para la pr ctica de AF de forma libre o dirigida. Sin embargo, el ambiente puede influir de manera importante en los niveles de AF. En este estudio se pudieron observar niveles de sedentarismo mucho $\mathrm{m}$ s altos que los observados en previos estudios (McKenzie, Marshall, SaIlis \& Conway, 2000; Anthamatten, Brink, Lampe, Greenwood, Kingston \& Nigg, 2011; Springer, Tanguturi, Ranjit, Skala \& Kelder, 2009). En la mayor a de estos se reportan niveles de MVPA mayores al $60 \%$ en ni os y adolescentes de 6 a 17 a os.

El ambiente de las escuelas $p$ blicas en Colombia es poco motivante para la pr ctica de la actividad f sica; lo reducido de los espacios disponibles para la AF durante el descanso se refleja en la gran densidad de poblaci $n$ escolar que se puede observar durante estos per odos. Los materiales con los que est n construidos las escuelas, en su mayor a cemento, pavimento, piedras y ladrillos, son poco amigables, y la falta de mantenimiento 
convierten a los patios escolares en sitios peligrosos porque las consecuencias de una ca da en un piso de pavimento o concreto desgastado pueden ser fatales. El alto nivel de ruido y contaminaci $\mathrm{n}$ del aire pueden ser tambi $\mathrm{n}$ factores que desmotivan para la pr ctica de la AF durante el descanso. La disponibilidad de implementos deportivos durante los descansos ha demostrado ser un factor de motivaci $\mathrm{n}$ para incrementar el nivel de actividad de los escolares (Verstraete et al., 2006). Sin embargo, la falta de espacio disponible caracter stica de las escuelas $p$ blicas en Colombia (hasta 700 ni os deben compartir un espacio de $20 \times 30 \mathrm{~m}$ ) convierte el uso de balones, cuerdas, aros, patines, etc. en un riesgo que la administraci $\mathrm{n}$ escolar y los docentes prefieren evitary, al contrario de lo recomendado (Anthamatten et al., 2011), proh ben el uso de estos implementos

A diferencia de otros estudios (Mota et al., 2005; Vilhjalmsson \& Kristjansdottir, 2003) en el presente estudio no se encontraron diferencias de $\mathrm{g}$ nero significativas en la proporci $\mathrm{n}$ de ni os o adolescentes que permanecen activos durante el descanso. Aunque los ni os fueron algo $\mathrm{m} \mathrm{s}$ activos que las ni as, las diferencias no son muy grandes. Los adolescentes masculinos tampoco muestran niveles de actividad mucho mayores que las adolescentes.

Diferencias significativas fueron encontradas al comparar los niveles de AF de ni os y adolescentes. Los ni os, siguiendo su impulso natural de movimiento, utilizan adem $\mathrm{s}$ del patio escolar otros ambientes no dise ados y menos propicios que el patio, como las escaleras y los corredores. Los adolescentes solo se muestran activos en el patio de la escuela y utilizan los dem $\mathrm{s}$ ambientes para actividades que no requieren $\mathrm{AF}$, como socializar e ingerir alimentos. El hecho de que los adolescentes disfruten de un per odo adicional de 20 minutos de descanso no influye sobre los niveles de AF, que son siempre inferiores en ambos g neros, siendo las ni as mucho $\mathrm{m}$ s activas que las adolescentes y los ni os mucho $\mathrm{m}$ s activos que los adolescentes. y adolescentes durante el descanso en la escuela, un estudio... 
El presente estudio se limita a registrar niveles de AF en una escuela a donde asisten ni os de bajos recursos econ micos. La falta de una observaci $n$ en escuelas de diferente estrato socioecon mico impide determinar si la variable ambiental es la causa principal de los bajos niveles de AF de ni os y adolescentes. En futuros estudios se deber an hacer comparaciones con ni os de estratos socio-econ micos medio y alto, para tratar de delimitar las causas de la desmotivaci $\mathrm{n}$ para el movimiento y aislar variables que podr an ser manipuladas en programas de promoci $\mathrm{n}$ de la AF en las escuelas.

Los responsables de pol ticas educativas deber an tener en cuenta la influencia directa de la planta $f$ sica de las escuelas, los materiales con los que son construidas y los espacios disponibles para el movimiento, sobre los niveles de AF de los ni os y adolescentes. Mejores instalaciones podr an constituir factor de motivaci n para que los ni os utilicen el descanso para tratar de acumular la mayor cantidad de minutos de AF de moderada a vigorosa y as superar las limitaciones para el movimiento que se presentan fuera de las escuelas en los barrios.

\section{CONCLUSIONES}

Menos de la mitad de los ni os de una escuela de bajo nivel socio-econ mico utilizan el per odo de descanso en la escuela para la pr ctica de AF de intensidad moderada a vigorosa. Los ni os de $b$ sica primaria de ambos $g$ neros son $m$ s activos que los adolescentes de secundar a.

AGRADECIMIENTOS: el autor agradece a los directivos, docentes y escolares de la escuela Rep blica del Uruguay por permitir el acceso a la instituci $n$ y por su participaci $n$ y contribuci $n$ al presente estudio. Reconocimiento a Carlos Alberto Ram rez, por su ayuda en el registro de im genes y proceso de datos. 


\section{REFERENCIAS}

1. Anthamatten, P., Brink, L., Lampe, S., Greenwood, E., Kingston, B., \& Nigg, C. (2011). An assessment of schoolyard renovation strategies to encourage children's physical activity. International Journal of Behavioral Nutrition and Physical Activity, 8(27), 2-9.

2. Blatchtford, P., Baines, E., \& Pellegrini, A. (2003). The social context of school playground games: sex and ethnic differences, and changes over time after entry to junior school. British Journal of Developmental Psychology, 21,481-505

3. Beighle, A., Morgan, C., LeMasurier, G., \& Pangrazi, R. (2006). Children's physical activity during recess and outside of school. J Sch Health. 2006, 76(10), 516-520.

4. Davison, K., \& Lawson, C. (2006). Do attributes in the physical environment influence children's physical activity? A review of the literature. Int J Behav Nutr Phys Act, 3(19).

5. De Saint-Maurice, F. (2009). Validation of the SOPLAY direct observation tool with an objective accelerometry-based physical activity monitor (Graduate Theses and Dissertations). USA: Iowa State University.

6. McKenzie, T. (2002). The use of direct observation to assess physical activity. In G. Welk (Ed.), Physical Activity Assessments for Health- Related Research (pp.179-195). Champaign, IL: Human Kinetics.

7. McKenzie, T., Baquero, B., Crespo, N., Arredondo, E., Campbell, N., \& Elder, J. (2008). Environmental correlates of physical activity in Mexican American children at home. J Phys Act Health, 5(4), 579-591.

8. McKenzie, T., Cohen, D., Sehgal, A., Williamson, S., \& Golinelli, D. (2006). System for Observing Play and Recreation in Communities (SOPARC): reliability and feasibility measures. J Phys Act Health, 1, S208-S222.

9. McKenzie, T., Marshall, S., Sallis, J., \& Conway, T. (2000). Leisure-time physical activity in school environments: an observational study using SOPLAY. Prev Med, 30, 70-77.

10. McKenzie, T., Sallis, J., \& Elder J. (1997). Physical activity levels and prompts in young children at recess: a two-year study of a bi-ethnic sample. Res Q Exerc Sport, 68(3), 195-202.

11. McKenzie, T., Sallis, J., \& Patterson, T. (1991). BEACHES: an observational system for assessing children's eating and physical activity behaviors and associated events. J Appl Behav Anal, 24, 141-151.

12. Mota, J., Suva, P., Santos, M., Ribeiro, J., Oliverira, J., \& Duarte, J. (2005). Physical activity and school recess time: differences between the sexes and the relationship between children's playground physical activity and habitual physical activity. J Sports Sci, 23(3), 269-275. 
13. Pangrazi, R. (2000). Promoting physical activity for youth. J Sci Med Sport 3(3), 280-286.

14. Pate, R., Davis, M., \& Robinson. T. (2006). Promoting physical activity in children and youth: a leadership role for schools. Circulation, (114)11, 1214-1224.

15. Ridgers, N., Stratton, G., \& McKenzie, T. (2010). Reliability and validity of the System for Observing Children's Activity and Relationships during Play (SOCARP). J Phys Act Health, 5, 17-25.

16. Ridgers, N., Stratton, G., Fairclough, S., \& Twisk, J. (2007). Long-term effects of a playground markings and physical structures on children's recess physical activity levels. Prev Med, 44(5), 393-397.

17. Rowlands, A. V., \& Hughes, D. R. (2006). Variability of physical activity patterns by type of day and season in 8-10 y old boys. Research Quarterly for Exercise and Sport, 77(3), 391-395.

18. Sarkin, J., McKenzie, T., \& Sallis, J. (1997). Gender differences in physical activity during fifth-grade physical education and recess periods. J Teach Phys Educ, (17), 99-106.

19. Springer, A., Tanguturi, Y., Ranjit, N., Skala, K., \& Kelder, S. (2009). Physical Activity During Recess in Low-Income Third-Grade Texas Students. Am J Health Behav, 37(3), 318-324.

20. Stratton, G., \& Mullan, E. (2005). The effect of multicolor playground markings on children's physical activity levels during recess. Prev Med, 41, 828-833.

21. USDHHS US Department of Health and Human Services \& USDA US Department of Agriculture (2005). Dietary Guidelines for Americans. Washington DC: The Authors.

22. USDHHS US Department of Health and Human Services (2008). Physical Activity Guidelines for Americans. Washington, DC: The Department.

23. Verstraete, S., Cardon, G., DeClercq, D., \& Bourdeaudhuij, I. (2006). Increasing children's physical activity levels during recess periods in elementary schools: the effects of providing game equipment. Eur J Pub Health, 16(4):415-419.

24. Vilhjalmsson, R., \& Kristjansdottir, G. (2003). Gender differences in physical activity in older children and adolescents: the central role of organized sport. Social Science \& Medicine, 56(2), 363-374.

25. Wechsler, H., Devereaux, R., Davis, M., \& Collins, J. (2000). Using the school environment to promote physical activity and healthy eating. Prev Med, (31), 121-137.

Recepción: 05-05-2013

Aprobación: 05-03-2014 